\title{
Decreasing excitation gap in Andreev billiards by disorder scattering
}

\author{
Florian Libisch,, , 丹 Jürgen Möller, ${ }^{1}$ Stefan Rotter, ${ }^{2}$ Maxim G. Vavilov, ${ }^{3}$ and Joachim Burgdörfer ${ }^{1}$ \\ ${ }^{1}$ Institute for Theoretical Physics, Vienna University of Technology, A-1040 Vienna, Austria, EU \\ ${ }^{2}$ Department of Applied Physics, Yale University, New Haven, CT 06520, USA \\ ${ }^{3}$ Department of Physics, University of Wisconsin, Madison, WI 53706, USA
}

(Dated: August 16, 2021)

\begin{abstract}
We investigate the distribution of the lowest-lying energy states in a disordered Andreev billiard by solving the Bogoliubov-de Gennes equation numerically. Contrary to conventional predictions we find a decrease rather than an increase of the excitation gap relative to its clean ballistic limit. We relate this finding to the eigenvalue spectrum of the Wigner-Smith time delay matrix between successive Andreev reflections. We show that the longest rather than the mean time delay determines the size of the excitation gap. With increasing disorder strength the values of the longest delay times increase, thereby, in turn, reducing the excitation gap.
\end{abstract}

PACS numbers: 74.45.+c, 73.21.La, 05.45.MT

A small metallic grain connected to a superconductor, commonly referred to as "Andreev billiard" (AB) [1], features very intriguing electron dynamics that has been the focus of numerous studies, both theoretical and experimental $[2,3,4,5,6,7,8,9,10,11,12,13,14,15,16$, 17, 18, 19, 20, 21, 22, 23, 24, 25, 26, 27, 28] (for a review see [29]). The energy spectrum in such a grain is strongly influenced by the process of Andreev reflection of quasi particles at the contact between the superconductor and the normal metal. At this "SN-interface" an incoming electron with energy $\varepsilon$ (counted from the Fermi energy $E_{F}$ ) is back-reflected as a "hole" with energy $-\varepsilon$ and nearly opposite momentum [1, 30]. Such Andreev reflections result in the coupling between electron and hole excitations in the normal metal resembling those of electron-hole states in superconductors. In particular, the density of states (DOS) near the Fermi edge $\left(E_{\mathrm{F}}\right)$ is reduced and may exhibit an "excitation gap" $\left(E_{1}\right)$. Details of this reduced DOS are determined by the dynamics in the Andreev billiard which, in turn, depends on the boundary geometry, the position of the SN interface and on the potential surface in the $\mathrm{AB}$. The distance $E_{1}$ of the first excited state in the grain ("billiard") from the Fermi level (set equal to zero in the following) marks the size of the excitation gap in the energy spectrum. While being much smaller than the bulk gap $\Delta$ of the superconductor, $E_{1} \ll \Delta$, $E_{1}$ may considerably exceed the mean level spacing $\delta$, i.e. the average energy distance between adjacent eigenstates, thus signalling the appearance of a gap.

Qualitative insights into the origin of spectral features in an Andreev structure, in particular the appearance of a gap, can be gained from a semiclassical analysis. The semiclassical Bohr-Sommerfeld (BS) approximation 10, 11, 15, 18, 27] allows to relate Andreev-reflected periodic orbits with the energy levels of the Andreev billiard [see Fig. 1(a)]. An energy eigenstate corresponds to a standing wave along such a periodic electron-hole

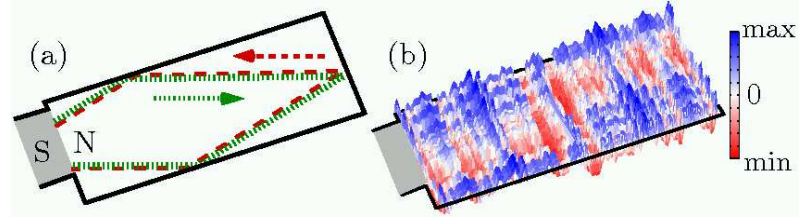

FIG. 1: (Color online) (a) Geometry of an Andreev billiard $(\mathrm{AB})$ consisting of a rectangular normal $(\mathrm{N})$ conductor of dimension $(1.5 W, 3 W)$, where $W$ is the width of the junction with the superconductor $(\mathrm{S})$. The dotted (dashed) line depicts the electron (hole) part of a periodic electron-hole orbit created by Andreev reflection at the SN-interface. (b) A sample realization of the landscape of the disorder potential inside $\mathrm{N}$.

orbit with the action difference between electron and hole being quantized. This simple picture implies that the lowest energy $E_{1}$ in the $\mathrm{AB}$ (i.e. the excitation gap) will be inversely proportional to the length of the longest electron-hole trajectories. However instructive the BS approach may be, it suffers from serious limitations, resulting from the assumption of strictly retracing electron-hole orbits. Corrections are due to short-range scattering off disorder [4, 9, 14, 23], quantum diffraction [11, 16, 19, 22, 23, 28], deviations of the Andreev reflection from perfect backscattering [22, 28] as well as due to contributions from trajectories that do not couple to the SN-interface [18, 28]. These corrections may turn out to be so large as to render a prediction for the excitation gap based on the BS approach unreliable. For example, the formation of a sizeable excitation gap in chaotic Andreev billiards as predicted by Random Matrix Theory (RMT) and verified numerically [10], is not reproduced by the BS approximation [13].

In a realistic metal sample brought into contact with a superconductor, deviations from the ballistic limit by disorder scattering play an important role. If the elastic mean free scattering path $\ell$ is smaller than the linear dimension of the metal grain, the trajectory between 


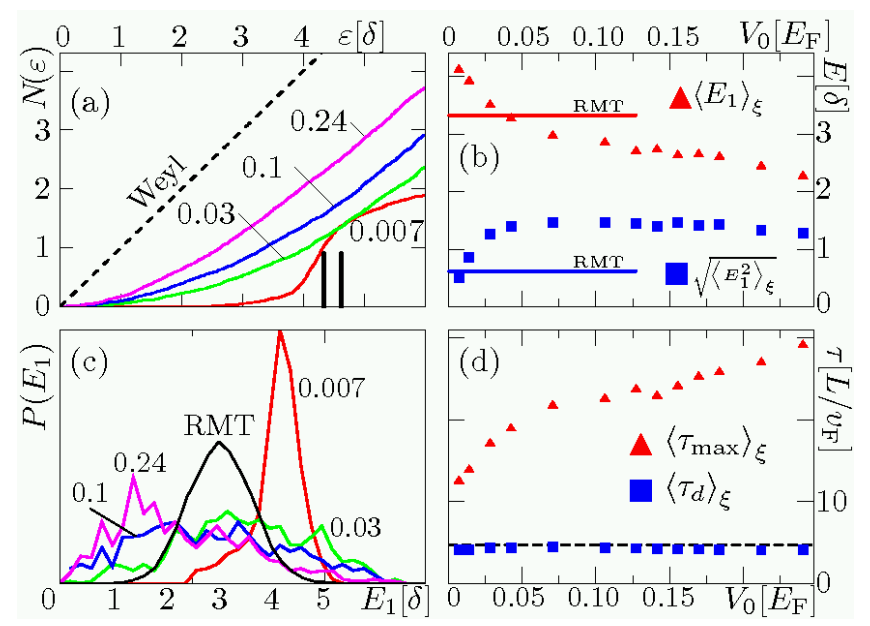

FIG. 2: (Color online) (a) Disorder-averaged state counting function, $\langle N(\varepsilon)\rangle_{\xi}$, for four different disorder strengths $V_{0} / E_{F}=0.007,0.03,0.1$ and 0.24 (colored solid lines) and Weyl estimate (black dashed line). The two lowest energy eigenvalues of the disorder-free system are marked by vertical bars. (b) Evolution of the mean gap $\left\langle E_{1}\right\rangle_{\xi}$ (red triangles) and the root mean square deviation (blue squares) as a function of disorder strength $V_{0}$ (in units of $E_{\mathrm{F}}$ ). Horizontal lines mark the RMT predictions. (c) Statistical distribution of the lowest eigenvalue $E_{1}$ for four disorder strengths $V_{0}$ (colored lines) compared with the RMT distribution (black line). (d) Dependence of the Wigner-Smith delay times on disorder strength $V_{0}$. Both the mean delay time, i.e., the dwell time $\left\langle\tau_{\mathrm{d}}\right\rangle_{\xi}$ (blue squares), and the maximum delay times $\left\langle\tau_{\max }\right\rangle_{\xi}$ (red triangles) are shown. The black dashed line shows the estimate $\left\langle\tau_{d}\right\rangle_{\xi}=2 \pi / N \delta^{\prime}$ from 32].

two successive Andreev reflections at the SN-interface is dominated by disorder scattering in the interior of the grain rather than by ballistic scattering off the grain boundaries. It has been suggested that the shortening of electron-hole orbits or, equivalently, of the average dwell time $\tau_{\mathrm{d}}$ between successive Andreev reflections by disorder scattering would lead to an increase of the excitation gap as compared to that of a clean SN junction [3, 14]. Such a trend would qualitatively be in line with recent investigations 23. which have found that the gap in the ensemble averaged density of states of an $\mathrm{AB}$ increases as the mean free path decreases with respect to the clean, ballistic limit (under the assumption of constant average dwell times $\tau_{\mathrm{d}}$ ).

In this letter, we present numerical ab initio simulations for a two-dimensional $\mathrm{AB}$ with disorder, employing the Modular Recursive Green's function Method (MRGM) in combination with a wave-function matching technique at the SN interface 27, 31]. Disorder is represented by elastic scattering off a potential distribution with short-range disorder with a correlation length $l_{V}$ small compared to the Fermi wavelength, $l_{V} / \lambda_{F}=0.12$. Decohering processes such as inelastic scattering are neglected in the following.
We choose a rectangular normal(N)-conducting cavity with dimensions $(1.5 \mathrm{~W}, 3 \mathrm{~W})$ where $W$ is the width of the superconducting lead [see Fig. 1(a)]. We construct the disorder potential [Fig. 1(b)] by decomposing the N region into two quadratic modules of dimension $(1.5 \mathrm{~W}$, $1.5 \mathrm{~W}$ ) within each of which we employ a separable random potential, $V_{\xi}(x, y)=V_{\xi_{x}}(x)+V_{\xi_{y}}(y)\left[\xi_{x}, \xi_{y}\right.$ denote two different statistical samples, jointly refereed to as $\left.\xi \equiv\left\{\xi_{x}, \xi_{y}\right\}\right]$. This "trick" is employed for reasons of numerical efficiency, in particular for small $\lambda_{\mathrm{F}}$ [31]. We ensure truly random scattering by destroying any unwanted separability by rotating by $180^{\circ}$ the random potentials in the two squares relative to each other [see Fig. 1(b)]. The spatial correlation of the random potential is characterized by $\left\langle V_{\xi}(x, y) V_{\xi}(x+a, y)\right\rangle_{x, y}=\left\langle V_{\xi}(x, y) V_{\xi}(x, y+\right.$ $a)\rangle_{x, y}=V_{0}^{2} \times \exp \left(-a / l_{V}\right)$, with $\langle\cdots\rangle_{x, y}$ indicating a spatial average over the whole disorder area and $l_{V}$ the correlation length. For a given realization $\xi$ the potential has zero spatial average, $\left\langle V_{\xi}(x, y)\right\rangle_{x, y}=0$, and an amplitude, $V_{0}=\sqrt{\left\langle\left[V_{\xi}(x, y)\right]^{2}\right\rangle_{x, y}}$, which is chosen to be small compared to the Fermi energy, $V_{0} / E_{F} \lesssim 0.2$. For $V_{0} \rightarrow 0$ the dynamics in the normal conducting part of the $\mathrm{AB}$ is entirely ballistic (no disorder scattering) and regular (due to the rectangular confinement). Calculations are performed with $N=24$ open transverse modes fitting in the lead width $W$ of the superconductor. The SNinterface itself is assumed to be ideal (no tunnel barrier) and the superconducting coherence length is assumed to be smaller than any other length parameters in the cavity. The superconducting gap $\Delta$ was chosen as $0.2 E_{\mathrm{F}}$ to ensure that the energy $E_{1}$ of the lowest-lying eigenstate fulfills $E_{1} \ll \Delta$.

In the fully ballistic limit, i.e., in the absence of any disorder, $V_{0}=0$, we find the lowest energy $E_{1}$ to be four times larger than the AB's mean level spacing $\delta$ [see Fig. 2(a) for the lowest eigenenergies]. To investigate the influence of the disorder on the energy spectrum we now gradually increase the disorder amplitude $V_{0}$. For each value of $V_{0}$ we calculate the full energy spectrum (below the superconducting gap $\Delta$ ) for 500 different disorder realizations $\xi$, and determine the ensemble averaged state-counting function $N(\varepsilon)$ (i.e., the integrated DOS) in the ensemble-average. For very weak disorder strength, $V_{0} / E_{\mathrm{F}}=0.007$, we find $\langle N(\varepsilon)\rangle_{\xi}$ to be still very close to the fully ballistic limit of $V_{0}=0$ where the spectral density close to $E_{F}$ is strongly suppressed relative to the Weyl estimate $N(\varepsilon)=\rho \varepsilon$ for the DOS per unit area $\rho=m_{\text {eff }} /\left(\pi \hbar^{2}\right)$ [see Fig. 2(a)]. Increasing the disorder amplitude $V_{0}$, however, gradually shifts $N(\varepsilon)$ towards the Weyl distribution [see Fig. 2(a,b)]. In particular, we find the size of the excitation gap to be reduced with increasing values of $V_{0}$, rather than increased. The reduction of $E_{1}$ is a sizeable (factor 2 in the range $0 \leq V_{0} / E_{\mathrm{F}} \leq 0.2$ ) and robust effect. For comparison we also show the gap as predicted by RMT for chaotic systems [see Fig. 2(b)]. These RMT estimates are based on a numerical calcu- 
lation representing the internal dynamics of the normal conductor in the $\mathrm{AB}$ by an ensemble of 8000 symmetric random matrices of size $M \times M$ [17]. $M$ is assumed to scale with the ratio of cavity circumference $C$ to the size of the SN junction $W, M=N \times C / W$ (for $N=24$ modes in the SN-interface we obtain $M=216$ ). While the RMT value of the gap, $E_{1} \approx 3.28 \delta$, is in reasonable agreement with our numerical data for finite disorder strength $V_{0} \neq 0$, significant discrepancies appear for the second moment (i.e. the variance) of the distribution $\sqrt{\left\langle E_{1}^{2}\right\rangle_{\xi}}$ [see Fig. 2(b,c)]. The full quantum calculation shows first a steep increase in the variance with increasing disorder strength before levelling off, whereas the RMT result underestimates the width of the distribution drastically. It should be noted that, strictly speaking, the limit of universality is only expected to hold for $M \gg N \gg N^{1 / 3} \gg 1$ [10]. The latter limit is difficult to reach in any realistic simulation for a two-dimensional cavity. The fact that both the gap size and the variance stay at an almost constant value in a whole interval of the disorder strength, $0.1 \leq V_{0} / E_{F} \leq 0.2$, possibly points to a saturation effect due to the disorder-induced randomization of otherwise boundary-specific scattering dynamics. The reduction of gap size and variance for stronger disorder, $V_{0} / E_{\mathrm{F}}>0.2$, may be related to a transition from weakly disordered scattering to diffusive or localized dynamics.

The strong reduction of the gap size with increasing disorder points to a mechanism qualitatively different from the behaviour of the mean dwell time $\left\langle\tau_{\mathrm{d}}\right\rangle_{\xi}$, which is only negligibly affected by increasing disorder [see Fig. 2(d)]. To uncover the underlying physics we employ a rigorous approach that allows to relate the energy spectrum of a quantum system to the dwell time distribution that does invoke neither any semiclassical approximation nor random matrix assumptions. Key to our approach is the relation between the Wigner-Smith (WS) time delay matrix $Q$ and the scattering matrix $\mathrm{S}[33, \underline{34}$,

$$
Q(\varepsilon)=-i \hbar S^{\dagger}(\varepsilon) \partial S(\varepsilon) / \partial \varepsilon .
$$

Equation (11), well-known for unbound quantum systems, can be applied to the (bound) spectrum of an $\mathrm{AB}$ since an eigenstate of the $\mathrm{AB}$ occurs at an energy $\varepsilon$ for which the determinant [5]

$$
\operatorname{det}\left[1+S(\varepsilon) S^{\dagger}(-\varepsilon)\right]=0,
$$

where $S(\varepsilon)$ is the scattering matrix of the open, normalconducting cavity with the superconductor replaced by a normal conducting waveguide of identical width $W$. Expanding $S(\varepsilon)$ around the Fermi energy $(\varepsilon=0)$ for small $\varepsilon$ yields

$$
S(\varepsilon) S^{\dagger}(-\varepsilon)=\mathbb{1}+2 \frac{i}{\hbar} \varepsilon Q+\ldots \approx \exp \left(2 \frac{i}{\hbar} \varepsilon Q\right),
$$
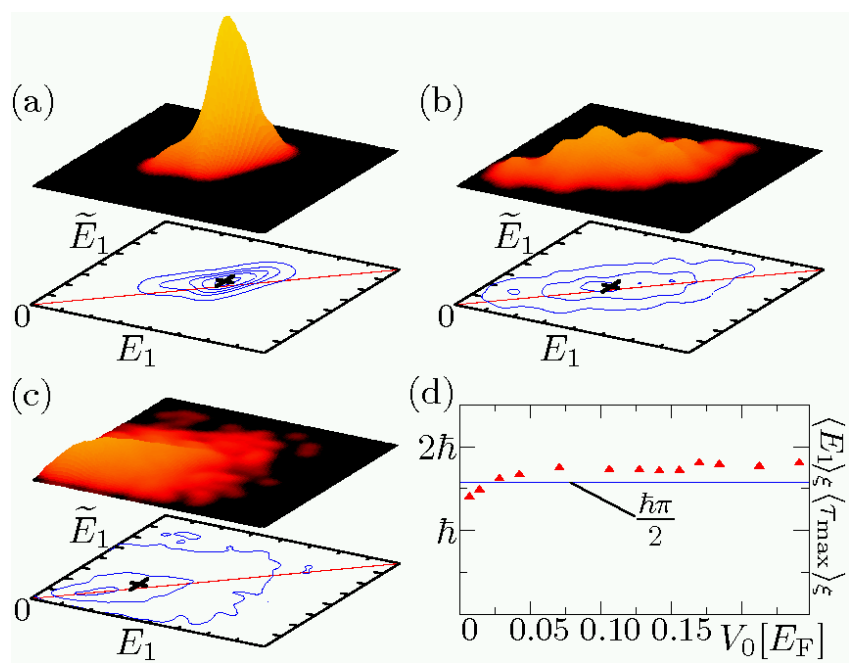

FIG. 3: (Color online) Smoothed distribution of $\left(E_{1}, \widetilde{E}_{1}\right)$ pairs for three different strengths of the disorder potential,(a) $V_{0} / E_{\mathrm{F}}=0.007$, (b) 0.03 , (c) 0.24 (500 realizations of disorder were used). Black crosses in the contour plot mark the mean value of the distribution. Perfect $\left(E_{1}, \widetilde{E}_{1}\right)$ correlation would correspond to non-vanishing density only along the diagonal (drawn in the contour plot as guide to the eye). (d) Product of the mean gap size $\left\langle E_{1}\right\rangle_{\xi}$ and the mean of the maximum Wigner-Smith delay time $\left\langle\tau_{\max }\right\rangle_{\xi}$ as a function of disorder strength. The constant value $\hbar \pi / 2$ [predicted by Eq. (5)] is shown for comparison.

and, in turn, the approximate quantization condition for Andreev states [21]:

$$
1+\exp \left(2 \frac{i}{\hbar} \varepsilon \tau_{n}\right)=0
$$

The Wigner-Smith delay times $\tau_{n}$ are the eigenvalues of $Q$. They correspond to "sticking" times inside the normal-conducting cavity between entering and leaving the cavity through the opening. Since in an AB the opening is replaced by an $\mathrm{SN}$ junction, $\tau_{n}$ measures the dwell-time between two successive Andreev reflections.

The values of $\tau_{n}$ (with $n \leq N$ ) provide a basisindependent measure for the sticking time of "quantum trajectories" without invoking model-specific assumptions or semiclassical approximations. The only limitation of Eq. (4) is the error of order $O\left(\varepsilon^{2}\right)$ due to the Taylor expansion and approximate resummation of the unitary operator $S(\varepsilon) S^{\dagger}(-\varepsilon)$ [Eq. (3)]. Equation (4) relates the energy spectrum at small $\varepsilon$ to the largest delaytime eigenvalues. In particular, the size of the excitation gap $E_{1}$ is determined by the maximal delay time value $\tau_{\max }$, such that

$$
E_{1} \approx \frac{\hbar \pi}{2 \tau_{\max }} \equiv \widetilde{E}_{1}
$$

The disorder-averaged maximum delay time, $\left\langle\tau_{\max }\right\rangle_{\xi}$, is, indeed, monotonically increasing with increasing disorder 
strength $V_{0}$ [Fig. 2(d)]. In turn, Eq. (5) suggests that the disorder-averaged gap $\left\langle E_{1}\right\rangle_{\xi}$ will be reduced.

To probe for the correlation between maximum delay time $\left(\tau_{\max }\right)$ and the gap size $\left(E_{1}\right)$ hinted at by Eq. (5), we have performed a statistical analysis of the distribution of $\left(E_{1}, \tau_{\max }\right)$ pairs for 500 disorder realizations (Fig. 3), converted to $\left(E_{1}, \widetilde{E}_{1}\right)$ pairs using Eq. (5). For perfect correlation we should expect the histogram to feature non-zero bins only along the diagonal $\left(\widetilde{E}_{1}=E_{1}\right)$. Deviations from a perfect correlation, resulting in part from the Taylor expansion Eq. (3), provide a measure for the accuracy of the estimate $\widetilde{E}_{1}$ as compared to the exact gap size $E_{1}$. For small disorder strength $\left(V_{0} / E_{\mathrm{F}}=0.007\right)$ the correlation between $E_{1}$ and $\widetilde{E}_{1}$ is, indeed, near-perfect and non-zero bins occur only in a very limited range of values $E_{1}, \widetilde{E}_{1}$ [see Fig. 3(a)]. With increasing disorder strength $\left[V_{0} / E_{\mathrm{F}}=0.03\right.$, see Fig. 3(b)] the maximum in the distribution shifts to smaller values of $E_{1}$ and $\widetilde{E}_{1}$ while remaining correlated near the diagonal. Both observations underscore that increased disorder decreases the gap which is, indeed, correlated with the maximum WS time-delay eigenvalue. For much stronger disorder $\left[V_{0} / E_{\mathrm{F}}=0.24\right.$, see Fig. [3(c)], the $\left(E_{1}, \widetilde{E}_{1}\right)$ correlation is diminished as off-diagonal bins become more significantly populated. While, on average, the connection between the disorder-induced reduction of the gap and the increase of the maximal delay time $\left\langle\tau_{\max }\right\rangle_{\xi}$ still holds, see Fig. 3(d), for individual strong disorder realizations this picture breaks down and small (large) gap sizes may well occur for systems with small (large) values of $\tau_{\max }$.

The present simulations allow to directly inspect the effect of disorder scattering on the wavefunction densities in the particle and hole sectors. The latter provide a microscopic picture of the decay of correlations between gap and maximum delay time. In the ballistic limit $V_{0}=0$, the electron and hole wavefunction tend to closely mirror each other [Fig. [4(a)] in agreement with retracing electron and hole orbits between two Andreev re-

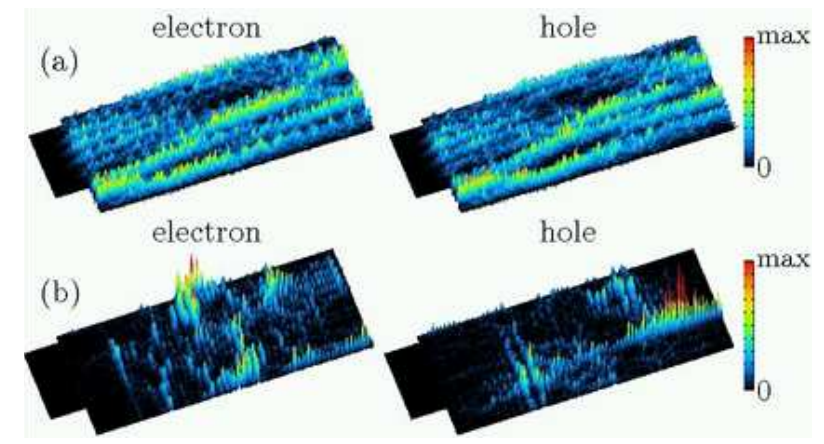

FIG. 4: (Color online) Electron and hole probability densities of the lowest Andreev eigenstate at (a) zero disorder potential, (b) finite disorder strength $V_{0} / E_{\mathrm{F}}=0.15$ (one disorder realization).

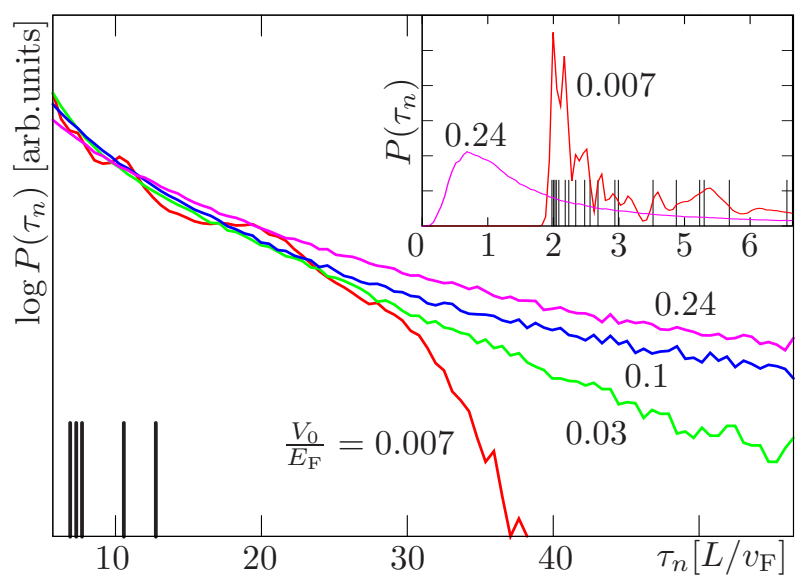

FIG. 5: (Color online) Distribution of Wigner-Smith delay times, $P\left(\tau_{n}\right)$ (colored lines) for an ensemble average over 500 disorder realizations $\xi$ and different disorder strength $\left(V_{0} / E_{F}=0.007,0.03,0.1,0.24\right)$. For each $\xi$ and $V_{0}$, the delay times were evaluated at 135 different energies in an interval $E_{\mathrm{F}} \pm 0.1 \Delta$. Increasing the disorder strength $V_{0}$ amplifies the long time tail of $P\left(\tau_{n}\right)$ (main plot, logarithmic scale), but concurrently produces much shorter delay times (see top right inset). The disorder-free delay times are indicated by vertical bars, with the lowest values starting at $\tau \approx 2 L / v_{F}$ (the time of flight across the cavity length $L=3 W$ and back) and the largest value at $\tau \approx 13 L / v_{F}$.

flections. With increasing disorder the similarity between wave components in the electron and hole sheet gradually disappears [see Fig. 4(b)]. This observation supports the picture that for strong disorder the wave function of the lowest $\mathrm{AB}$ eigenstate is largely determined by disorder scattering in the interior rather than by Andreev reflections at the SN interface. Accordingly, the dwell time between two Andreev reflections looses significance.

It is now instructive to inquire into the origin of the discrepancy to those models suggesting that the presence of disorder induces an increase rather than a decrease of the gap in comparison to its clean, ballistic limit [3, 14]. The key here is the disparate behaviour of the maximum, $\left\langle\tau_{\max }\right\rangle_{\xi} \equiv\left\langle\max _{n=1, N} \tau_{n}\right\rangle_{\xi}$, and average dwell time, $\left\langle\tau_{d}\right\rangle_{\xi} \equiv\left\langle\sum_{n}^{N} \tau_{n} / N\right\rangle_{\xi}$, the latter of which enters [3, 14]. For the system under study here, disorder scattering is obviously able to "delay" for long-lived scattering states the interval between two successive Andreev reflections. The presence of disorder not only increases $\left\langle\tau_{\max }\right\rangle_{\xi}$, but also reduces the minimal delay time $\left\langle\tau_{\min }\right\rangle_{\xi}$ (see Fig. 巸. As a consequence, the distribution of delay times $P\left(\tau_{n}\right)$ becomes "stretched", while leaving the mean value $\left\langle\tau_{d}\right\rangle_{\xi}$ almost unchanged. The fact that the average dwell time $\left\langle\tau_{d}\right\rangle_{\xi}$ stays almost unaffected by the increasing disorder [see Fig. 2(d)] is in agreement with a general relation 32] between the averaged trace of the matrix Q [see Eq. (11)], $\langle\operatorname{tr} Q\rangle_{\xi}$, and the mean spacing $\delta^{\prime}$ of resonant levels in a (normal-conducting) scattering system, $\langle\operatorname{tr} Q\rangle_{\xi}=2 \pi / \delta^{\prime}$ 
or, equivalently, $\left\langle\tau_{d}\right\rangle_{\xi}=\pi / N \delta$ (note that $\delta^{\prime}=2 \times \delta$, with $\delta$ being the mean level spacing in the corresponding AB). Consequently, the mean dwell time should be entirely independent of the disorder configuration. This is, indeed, very accurately confirmed by our numerical results for $\left\langle\tau_{d}\right\rangle_{\xi}$ [see Fig. 2(d)]. In turn, $\left\langle\tau_{d}\right\rangle_{\xi}$ is unsuitable for characterizing correlations between gap size and disorder strength, because of its independence of $V_{0}$. Therefore, relating the gap size to the mean dwell time $\left\langle\tau_{d}\right\rangle_{\xi}$ also fails to account for the gap reduction observed here [35]. Clearly, the present results do not preclude an increase of the excitation gap with increasing disorder for particular boundary shapes, e.g. for a gapless excitation spectrum in the absence of disorder. The present analysis suggests, however, that also in such systems the behaviour of the longest WS time delay eigenvalue will control the behaviour of the gap.

The results in Fig. [5]demonstrate that for a disordered cavity the strength of the disorder $\left(V_{0}\right)$ does have a crucial influence on the distribution of delay times $P\left(\tau_{n}\right)$ (in particular for long times). For chaotic cavities it was found both classically 36 and quantum mechanically [10, 31, 37] that the long time tail of delay times does not depend on certain system-specific parameters as, e.g., the Lyapunov exponent. We therefore expect that the statistical distribution of the gap size undergoes a crossover between the present case of a disordered $\mathrm{AB}$ and the case of a chaotic AB. It would be interesting to study such a crossover numerically, e.g., by tuning the disorder correlation length $l_{V}$ from the diffractive limit of $l_{V} \ll \lambda_{F}$ to the ballistic (chaotic) limit of $l_{V} \gg \lambda_{F}$.

With the help of Fig. 5 , we can furthermore explain the loss of correlations among pairs $\left(E_{1}, \widetilde{E}_{1}\right)$ for strong disorder [Fig. 3(c)]: The amplification of the maximal proper delay times $\left\langle\tau_{\max }\right\rangle_{\xi}$ by the increasing disorder translates into an increase of the high frequency components in the elements of the scattering matrix $S(\varepsilon)$. As, however, the estimate of the gap size, $\widetilde{E}_{1}$, relies in part on a Taylor expansion of $S(\varepsilon)$ [see Eq. (3)] which can only capture weakly energy dependent (i.e., low-frequency) components, the accuracy of $\widetilde{E}_{1}$ deteriorates with increasing disorder strength, thereby gradually diminishing the correlations among pairs $\left(E_{1}, \widetilde{E}_{1}\right)$. The behaviour of the mean values $\left\langle E_{1}\right\rangle_{\xi}$ and $\left\langle\widetilde{E}_{1}\right\rangle_{\xi}$ can be understood by considering the distribution of values $d_{E}=\left(E_{1}-\widetilde{E}_{1}\right) / 2$ (corresponding to a projection of the distributions of Fig. 3)(a-c) on an axis perpendicular to the diagonal $E_{1}=\widetilde{E}_{1}$ ). As we have verified numerically (not shown), the width of this distribution, $\operatorname{var}\left(d_{E}\right)=\sqrt{\left\langle d_{E}^{2}\right\rangle_{\xi}}$, increases with increasing $V_{0}$, while its mean value stays almost unaffected by the disorder strength at $d_{E} \ll \delta$. We speculate that the errors due to the Taylor expansion and the resummation of $S^{\dagger}(\varepsilon) S(-\varepsilon)$ [see Eq. (3)] are randomly distributed and thus cancel out in an average over many disorder realizations. This would explain why the averaged values $\left\langle E_{1}\right\rangle_{\xi}$ and $\left\langle\widetilde{E}_{1}\right\rangle_{\xi}=\left\langle\tau_{\max }^{-1}\right\rangle_{\xi}$ remain correlated [see Fig. 3(d)] while the correlation between individual pairs $\left(E_{1}, \widetilde{E}_{1}\right)$ breaks down.

In summary, we have numerically calculated the energy spectrum of electron-hole states in a rectangular Andreev billiard with a tunable disorder potential. In apparent contrast to qualitative models based on the mean cavity dwell time $\left\langle\tau_{\mathrm{d}}\right\rangle_{\xi}$, we find a decrease of the gap size when increasing the disorder amplitude. We show that this decrease is controlled by the disorder dependence of the largest Wigner-Smith delay time $\tau_{\max }$ between subsequent Andreev reflections at the SN-interface. The average dwell time $\left\langle\tau_{\mathrm{d}}\right\rangle_{\xi}$, on the other hand, only depends on the mean level spacing, and is thus neither correlated to the evolution of the gap size nor to the disorder scattering strength. Stronger disorder, however, drastically increases the value of $\tau_{\max }$ for long-lived scattering states. For sufficiently strong disorder the correlation between the gap size and $\tau_{\max }$ eventually breaks down for individual disorder realizations, as the eigenenergies of the system are then more strongly influenced by the specific disorder potential rather than by the Andreev reflection process.

We acknowledge helpful discussions with P. W. Brouwer (who also drew our attention to Ref. [32]), J. Feist and V. A. Handara. Support by the Austrian Science Foundation (FWF Austria, Grant No. FWFP17359), the Max Kade foundation (New York) and the W. M. Keck foundation is gratefully acknowledged.

* Electronic address: florian@concord.itp.tuwien.ac.at

[1] A. Andreev, Sov. Phys. JETP 19, 1228 (1964).

[2] D. Saint-James, J. Phys. (France) 25, 899 (1964).

[3] W. L. McMillan, Phys. Rev. 175, 537 (1968).

[4] A. A. Golubov and M. Y. Kuprianov, J. Low Temp. Phys. 70, 83 (1988).

[5] C. W. J. Beenakker, Phys. Rev. Lett. 67, 3836 (1991).

[6] I. Kosztin, D. L. Maslov, and P. M. Goldbart, Phys. Rev. Lett. 75, 1735 (1995).

[7] S. Guéron, H. Pothier, N. O. Birge, D. Estève, and M. H. Devoret, Phys. Rev. Lett. 77, 3025 (1996).

[8] A.Altland and M.Zirnbauer, Phys.Rev.Lett 76, 3420 (1996)

[9] W. Belzig, C. Bruder, and G. Schön, Phys. Rev. B 54, 9443 (1996).

[10] J. A. Melsen, P. W. Brouwer, K. M. Frahm, and C. W. J. Beenakker, Europhys. Lett. 35, 7 (1996); Phys. Scripta, T69, 223 (1997).

[11] A. Lodder and Y. V. Nazarov, Phys. Rev. B 58, 5783 (1998).

[12] N. A. Mortensen, K. Flensberg, and A.-P. Jauho, Physical Review B 59, 10176 (1999).

[13] H. Schomerus and C. W. J. Beenakker, Phys. Rev. Lett. 82, 2951 (1999).

[14] S. Pilgram, W. Belzig, and C. Bruder, Phys. Rev. B 62, 
12462 (2000) (see especially Figure 4).

[15] W. Ihra, M. Leadbeater, J. Vega, and K. Richter, Eur. Phys. J. B 21, 425 (2001).

[16] D. Taras-Semchuk and A. Altland, Physical Review B 64, 014512 (2001).

[17] M. G. Vavilov, P. W. Brouwer, V. Ambegaokar, and C. W. J. Beenakker, Phys. Rev. Lett. 86, 874 (2001).

[18] J. Cserti, A. Bodor, J. Koltai, and G. Vattay, Phys. Rev. B 66, 064528 (2002).

[19] I. Adagideli and C. W. J. Beenakker, Phys. Rev. Lett. 89, 237002 (2002).

[20] J. Cserti, A. Kormányos, Z. Kaufmann, J. Koltai, and C. J. Lambert, Phys. Rev. Lett. 89, 057001 (2002).

[21] M. G. Crawford and P. W. Brouwer, Phys. Rev. E 65, 026221 (2002).

[22] P. G. Silvestrov, M. C. Goorden, and C. W. J. Beenakker, Phys. Rev. Lett. 90, 116801 (2003).

[23] M. G. Vavilov and A. I. Larkin, Phys. Rev. B 67, 115335 (2003) (see especially Sec. III).

[24] M. C. Goorden, P. Jacquod, and C. W. J. Beenakker, Phys. Rev. B 68, 220501 (2003).

[25] A. Kormányos, Z. Kaufmann, J. Cserti, and C. J. Lambert, Phys. Rev. B 67, 172506 (2003).

[26] N. G. Fytas, F. K. Diabonos, P. Schmelcher, M. Scheid, A. Lassl, K. Richer, and G. Fagas, Phys. Rev. B 72, 085336 (2005).

[27] F. Libisch, S. Rotter, J. Burgdörfer, A. Kormányos, and J. Cserti, Phys. Rev. B 72, 075304 (2005).
[28] F. Libisch, S. Rotter, J. Burgdörfer, Phys. Rev. B 73, 045324 (2006).

[29] C. W. J. Beenakker, Lect. Notes Phys. 667, 131 (2005), (see especially chapters 3 and 6.3).

[30] A. A. Abrikosov, Fundamentals of the theory of metals (North-Holland, Amsterdam, 1988).

[31] S. Rotter, J.-Z. Tang, L. Wirtz, J. Trost, and J. Burgdörfer, Phys. Rev. B 62, 1950 (2000) (see especially Figure 12).

[32] V. L. Lyuboshitz, Phys. Lett. 72B, 41 (1977), Yad. Fiz. 27, 948 (1978) [Sov. J. Nucl. Phys. 27, 502 (1978)], Yad. Fiz. 37, 292 (1983) [Sov. J. Nucl. Phys. 37, 174 (1983)]

[33] E. P. Wigner, Phys. Rev. 98, 145 (1955).

[34] F. T. Smith, Phys. Rev. 118, 349 (1960).

[35] We emphasize here that throughout this letter, we have used the definition of the gap as the position of the lowest energy state in the $\mathrm{AB}$ above the Fermi energy (as in [17]). This definition should not be confused with the gap size in the average density of states, introduced for a large number of energy levels (as in [13, 23]). Also our definition of the dwell time as the mean of the Wignersmith delay times, $\left\langle\tau_{d}\right\rangle_{\xi}=\left\langle\frac{1}{N} \sum_{n=1}^{N} \tau_{n}\right\rangle_{\xi}$, should not be confused with other definitions used in the literature.

[36] W. A. Lin, J. B. Delos, and R. V. Jensen, Chaos 3, 655 (1993).

[37] P. W. Brouwer, K. M. Frahm, and C. W. J. Beenakker, Phys. Rev. Lett 78, 4737 (1997). 